\title{
What boxing tells us about repetitive head trauma and the brain
}

\author{
Charles Bernick* and Sarah Banks
}

\begin{abstract}
Boxing and other combat sports may serve as a human model to study the effects of repetitive head trauma on brain structure and function. The initial description of what is now known as chronic traumatic encephalopathy (CTE) was reported in boxers in 1928. In the ensuing years, studies examining boxers have described the clinical features of CTE, its relationship to degree of exposure to fighting, and an array of radiologic findings. The field has been hampered by issues related to study design, lack of longitudinal follow-up, and absence of agreed-upon clinical criteria for CTE. A recently launched prospective cohort study of professional fighters, the Professional Fighters Brain Health Study, attempts to overcome some of the problems in studying fighters. Here, we review the cross-sectional results from the first year of the project.
\end{abstract}

\section{Introduction}

It is not surprising that the long-term neurological consequences of cumulative head trauma were initially recognized in professional boxers [1]. These athletes are on the receiving end of thousands of blows to the head of varying intensity, in sparring and matches, over many years. Beginning in 1928, when Harrison Martland described the clinical features that constitute what is now known as chronic traumatic encephalopathy (CTE) [1], many articles have been written about the neurological consequences of boxing in both amateurs and professionals. Yet, there are still significant gaps in our knowledge of the spectrum of chronic injuries that can occur in combat sports.

It is worth asking what can we achieve by studying those in combat sports, both boxing and the increasingly popular sport of mixed martial arts (MMA). In the

*Correspondence: bernicc@ccf.org

Cleveland Clinic Lou Ruvo Center for Brain Health, 888 W. Bonneville Avenue, Las Vegas, NV 89106, USA broadest sense, these sports provide a human model to study the evolution of CTE, including understanding the natural history and modifying factors of the disease, along with identifying biomarkers. Thus, what is learned from combat sports may be applicable to various settings in which repetitive head trauma can occur, including other contact sports and the military. A more specific goal would be gathering knowledge that can be applied to improve long-term safety of boxing and MMA such as developing guidelines that can be used by regulatory agencies and the athletes themselves to better monitor their brain health.

In a review of the current body of literature on boxing and the brain, several caveats require mention. The sport itself has changed over the years, making comparison of studies from different decades difficult [2]. Current fighters tend to have shorter careers and fewer career bouts and benefit from rule changes such as limiting championship fights to 12 rounds (instead of 15), use of larger glove size, and increased medical supervision. Moreover, a number of methodological issues cloud the interpretation of prior work: (a) reliance on retrospective or crosssectional design, (b) lack of, or inadequate, control group, (c) evidence based on small sample sizes or case reports, and (d) selection bias of boxers who are symptomatic or have an extraordinarily high amount of exposure.

With the goal of overcoming the methodological limitations of prior research and addressing some of the important unanswered questions in the field of cumulative head trauma, we initiated a prospective cohort study of active and retired fighters in 2011, termed the Professional Fighters Brain Health Study (PFBHS). Whereas several excellent contemporary articles review the neurological effects of boxing, this article (a) will focus on how the current literature on fighters can inform us about the clinical and imaging features of CTE and (b) will describe the first-year results from the PFBHS [3-6]. For the purpose of this review, we will use the term CTE to subsume a number of terms used in the literature to denote chronic neurological findings in boxers, acknowledging that there is no way to know whether these individuals actually harbor the pathological changes we now attribute to CTE. 


\section{Review \\ Epidemiology}

A fundamental, but elusive, issue is just how common CTE is among those exposed to recurrent head trauma. In the absence of accurate clinical criteria or a large enough clinicopathological study group of symptomatic and asymptomatic individuals, this question cannot be answered satisfactorily. A commonly cited study of exprofessional fighters who were licensed to box in the UK from 1929 to 1955 found that 17\% of subjects had CTE and that $40 \%$ of the remaining boxers had disequilibrium, dysarthria, or alcoholism [7]. No methodologically sound studies of incidence or prevalence have been published since. On the other hand, the risk factors that have been consistently linked to chronic brain injury across prior work are older age and higher levels of exposure to head trauma [7-10].

Among the major challenges in the field of traumatic brain injury is being able to quantify exposure. In the absence of a direct measure of the cumulative trauma each subject is exposed to, several potential surrogates such as number of fights, fights per year, number of knockouts (KOs), and years of fighting have been used. However, each of these variables may actually have a slightly different influence on the development of CTE. Number of fights, for example, may act as a proxy for amount of training. Some have postulated that the effects of repeated blows to the head that occur during sparring, even at a subconcussive level, may play as important a role in causing cumulative brain injury as the match itself [11]. On the other hand, KOs may reflect the more severe end of the spectrum of mild traumatic brain injury. Whereas the number of KOs sustained in sanctioned professional fights can be tracked from commonly available records, the number of KOs that may have occurred at other times is harder to trace. Furthermore, frequency of fighting may be a complementary variable that requires consideration; fighting more frequently may reduce the time the brain has to fully recover from prior trauma and be a risk factor that interacts with number of fights.

Increased exposure to head trauma in and of itself does not appear to be sufficient to cause CTE. As in other neurodegenerative conditions, genetic factors may modify the risk of CTE. Some, but not all, studies have suggested that the apolipoprotein $\mathrm{E}_{4}$ allele increases the risk of Alzheimer's disease in individuals with a history of head trauma [12-14]. In a study of boxers, Jordan and colleagues [15] demonstrated an increased risk of CTE in those who are $E_{4}$-positive, although the study was retrospective in design.

\section{Clinical features}

A consistent picture of the clinical features of CTE in boxers has emerged over the years. However, whether these signs and symptoms develop in predictable stages is debated $[4,5,16]$. There does appear to be agreement that behavioral changes, ranging from affective disorders to paranoia, irritability, and aggression, occur frequently as an early symptom [4,5,9,17-19]. Progressively, cognitive dysfunction becomes noticeable with additional motor features such as dysarthria, parkinsonism, and gait disorder. These clinical observations in boxers are not too different from what was reported recently in a large clinicopathological study of athletes exposed to head trauma, in which headache, depression, and memory complaints were present in the early stages of CTE, followed by difficulties in gait and dysarthria (which was associated with motor neuron disease) and parkinsonism in the later stages [20]. The clinical information in that study was obtained retrospectively, and the informant may have reported only those symptoms and signs that were strikingly apparent. It is possible and perhaps likely that prospective and precise measurement of motoric and other neurological function reveals a slightly different sequence of signs and symptoms that occur with CTE associated with unarmed combat sports.

In regard to the specific neuropsychological domains effected in CTE, psychometric testing of former and active professional boxers has most frequently demonstrated deficits in memory, information-processing speed, finger-tapping speed, complex attentional tasks, and frontal-executive functions [5,9]. In contrast to professional fighting, amateur fighting has rarely been shown to result in any long-term changes in cognitive function [21]; longitudinal studies did not show any effect of boxing on psychometric results in amateurs even up to 9 years [22]. The use of psychometric measures as a means to screen for developing CTE in active fighters does have its hazards. Performance on any single testing session, particularly in proximity to a competition, can be influenced by a number of factors, including the acute effects of recent sparring, rapid weight loss and dehydration, pre-bout anxiety, and suboptimal effort. Moreover, the precision of psychometric tests used in this population may not be adequate to detect subtle changes given the variability of the tests themselves.

\section{Imaging}

Virtually every sort of imaging modality, ranging from pneumoencephalography to positron emission tomography (PET) scanning, has been studied in boxers [23]. Certainly, given its wide availability, lack of radiation exposure, and superior sensitivity over computed tomography imaging to detect subtle structural changes, magnetic resonance imaging (MRI) scanning has become the favored imaging modality for the evaluation of brain injury from head trauma. A number of MRI findings recognized by visual inspection have been related to 
boxing [24]. Several of these findings, including lateral ventricular size, dilated perivascular spaces, and diffuse axonal injury, were associated with some measure of exposure, such as number of professional bouts or years of fighting. Moreover, studies using measures of diffusivity on diffusion tensor imaging have shown changes at a group level between boxers and non-fighting groups [25-27].

Functional imaging has also been explored as a means of detecting brain injury that might not be seen on structural scanning. Studies employing single-photon emission tomography (SPECT) and PET imaging have reported differences between boxers and controls [28,29]. Despite a small sample size, there was a trend toward a relationship between number of fights and number and extent of PET abnormalities.

The application of what we know of imaging in fighters, at the moment, is limited. Most published imaging studies are cross-sectional and do not include a clinical outcome, so the significance of any one finding in predicting subsequent clinical change is unknown. In addition, the composition of the samples studied is usually not random, which may result in a bias of having more clincially symptomatic individuals participate. A number of other confounding factors in imaging studies have been reviewed by Moseley [23]. Although many states require MRI scans as part of licensing (and some states require the imaging to be repeated periodically), there is actually little evidence from well-designed studies to determine how to use the information to make decisions on fitness to fight or the value of these measures in protecting fighter safety.

\section{Professional Fighters Brain Health Study}

The PFBHS is a longitudinal study of active professional fighters (boxers and MMA fighters), retired professional fighters, and age/education-matched controls. The main objective of the PFBHS is to determine the relationships between measures of head trauma exposure, along with other potential modifiers, and changes in brain imaging and neurological/behavioral function over time. The study is designed to extend a minimum of 5 years, and an enrollment of more than 400 boxers and mixed martial artists is projected. Participants undergo annual evaluations to include 3-T MRI scanning, computerized cognitive assessments, speech analysis, surveys of mood and impulsivity, and blood sampling for genotyping and exploratory biomarker studies. Information is collected on demographics, educational attainment, family and medical history, previous head trauma (whether related or unrelated to athletic activities), prior involvement in other contact sports, and their amateur fighting history. The fighters' professional record is obtained from commonly cited websites (boxrec.com [30] for boxers and mixedmartialarts.com [31] and sherdog.com [32] for MMA fighters) to determine number of years of professional fighting, number and outcome of professional fights, number of rounds fought, weight class of each fight, frequency of professional fighting, and number of times knocked out (KOs and technical KOs). A composite fight exposure index was developed as a summary measure of cumulative traumatic exposure [33].

Several cross-sectional analyses have been performed on the baseline data obtained from the PFBHS to examine the association between fight exposure and various imaging measures. Repeated measures analysis of variance was employed to test the association between the outcome variables and fight exposure variables. Guided by the cutpoints (that is, tree branch splitting values) and deviance reduction values from the regression trees, we defined and tested fight exposure as follows: linear effect of total number of professional fights, linear effect of total number of years of professional fighting, a threshold effect with brain volume reduction estimated separately for less than 5 years of professional fighting versus at least 5 years, and an exposure composite score as a function of number of professional fights and number of professional fights per year. In each model, we included the type of fighter (boxer or MMA fighter) and an interaction term for the type of fighter with the other exposure variable. Given the exploratory nature of this study, a significance level of 0.05 was used to test the significance of the regression coefficients of the exposure variables; no adjustments for multiplicity were applied. A secondary aim was to test for associations between imaging measures and cognitive test scores and between fight exposure and cognitive test scores. Generalized linear models were constructed with cognitive scores as the dependent variables and brain volume or fight exposure variables as the independent variables of interest. All analyses were adjusted for age (treated as a continuous variable), education (defined as no college-level versus some college-level), and race, which was defined as (a) Caucasian, (b) AfricanAmerican, or (c) other (Asian, Pacific Islander, American Indian, or Alaskan Native).

Results from the baseline evaluations revealed findings that support and extend previously published work. Complete data on 239 subjects - 104 boxers and 135 MMA fighters - are currently available. The fighters' ages ranged from 19 to 43 , and the median was 28.3 years. Close to $52 \%$ of the subjects had a high school education or less, and $48.2 \%$ had at least some college-level education. The mean total number of years of professional fighting was 4 , and the median total number of professional fights was 11 (Table 1).

As might be expected, increasing exposure to head trauma, as measured by either number of professional 
Table 1. Demographic and fight exposure details of fighters recruited to date

\begin{tabular}{lccc}
\hline & Boxers & Mixed martial artists & Total \\
\hline Number & 104 & 135 & 239 \\
Age, years & $28.38(8.07)$ & $28.14(5.01)$ & $28.25(6.51)$ \\
Percentage of Hispanics & 46.05 & 27.68 & 34.76 \\
Percentage of Caucasians & 20.43 & 25.17 & 22.59 \\
Percentage of African-Americans & 53.76 & 19.93 & 39.42 \\
Percentage of Asians/Pacific Islanders & 3.23 & 4.55 & 3.97 \\
Years of education & $12.61(2.24)$ & $13.90(2.27)$ & $13.34(2.34)$ \\
Years of fighting & $3.96(4.95)$ & $4.08(3.98)$ & $4.03(4.42)$ \\
Number of fights & $11.85(16.54)$ & $10.56(13.05)$ & $11.11(14.62)$ \\
\hline
\end{tabular}

Values are presented as mean (standard deviation) unless otherwise indicated.

fights or years of professional fighting, was associated with lower volumes of several brain regions. Perhaps the most consistent relationship between exposure variables and brain volume was seen in the caudate and, less so, in the putamen [34]. Interestingly, for caudate and amygdala volumes, there was no effect of increasing number of years of professional fighting up to 5 years. However, above 5 years, there was a $1 \%$ reduction in caudate volume per additional year of professional fighting $(P<0.001)$ (Figure 1$)$. This raises the possibility that the relationship between fight exposure and reduction in brain volume is not linear; one might predict that a sequence of pathophysiologic changes occurs with repeated head trauma and that actual drop-out of neurons (and thus reduced volume) comes in a delayed fashion.

Similar associations between exposure and MRI measures of diffusivity and resting-state connectivity are seen. Like previous investigators, we found a significant relationship between number of fights and mean diffusivity values in the posterior corpus callosum. In addition, the number of times a fighter has been knocked out in his career predicted increased longitudinal and transverse diffusivity in white matter and subcortical gray matter regions including corpus callosum, cingulate, pericalcarine, precuneus, and amygdala, leading to increased mean diffusivity and decreased fractional anisotropy values in the corresponding regions in ROI analysis [35]. Preliminary analysis of resting-state functional MRI from a left posterior cingulate cortex seed showed that greater number of fights and KOs was associated with more impairment in the functional connectivity in anterior cingulate and cingulate gyrus (Figure 2).

Early results from a limited computerized cognitive battery found that only speed of processing was related to volume and exposure. Decreasing volumes of the thalamus, amygdala, left caudate, and hippocampus were associated with lower scores on speed of processing measures (Figure 3). On the other hand, processing speed

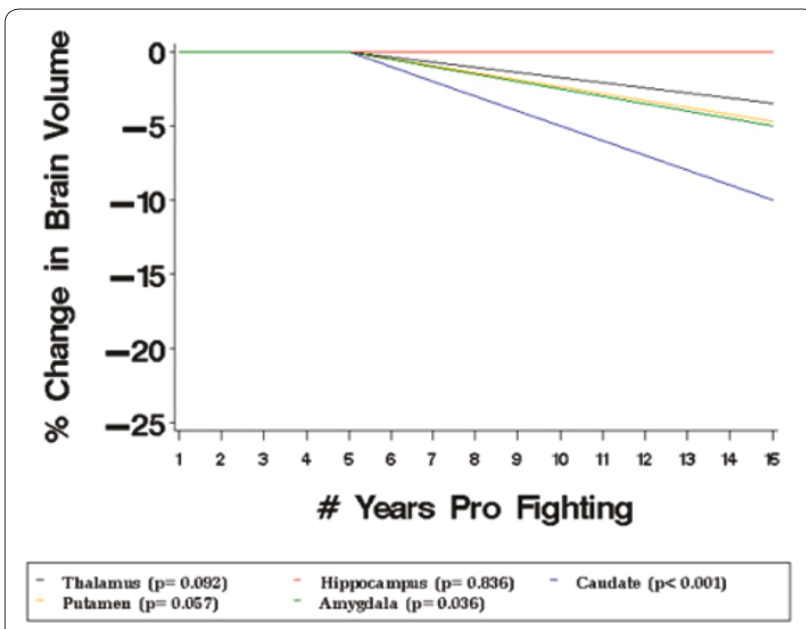

Figure 1. Estimated percentage change in brain volumes after adjustment for age, education, and race. Volumes are plotted against total years of professional fighting.

was related to exposure to head trauma only at the extremes of exposure. The fact that the association between exposure and processing speed was seen only between highest and lowest quartile is consistent with what is seen in other neurodegenerative diseases; the clinical expression of underlying pathology may not appear in a measurable way until a substantial amount of structural damage has occurred.

Level of education may have a modifying effect on the relationship between exposure and structural and cognitive changes. In the PFBHS, fighters with a high school education or less showed negative associations between fight exposure (number of fights and years of fighting) and cognitive tests scores (Figure 4). The relationship between brain structure volume and exposure did not differ on the basis of education. These results are interpreted as putatively showing a protective effect of education on functional, but not structural, integrity in fighters. 


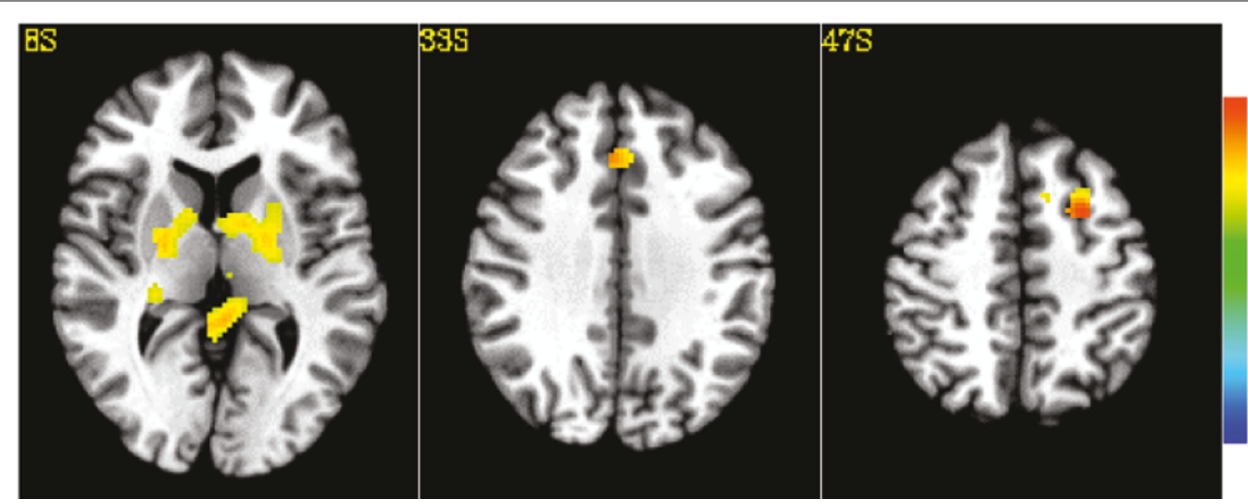

Figure 2. Areas where composite index predicts decreasing connectivity from the left poster cingulated cortex seed in the fighter population $(P<0.05, n=161)$. T score is presented with a color scale from -6 to +6 .

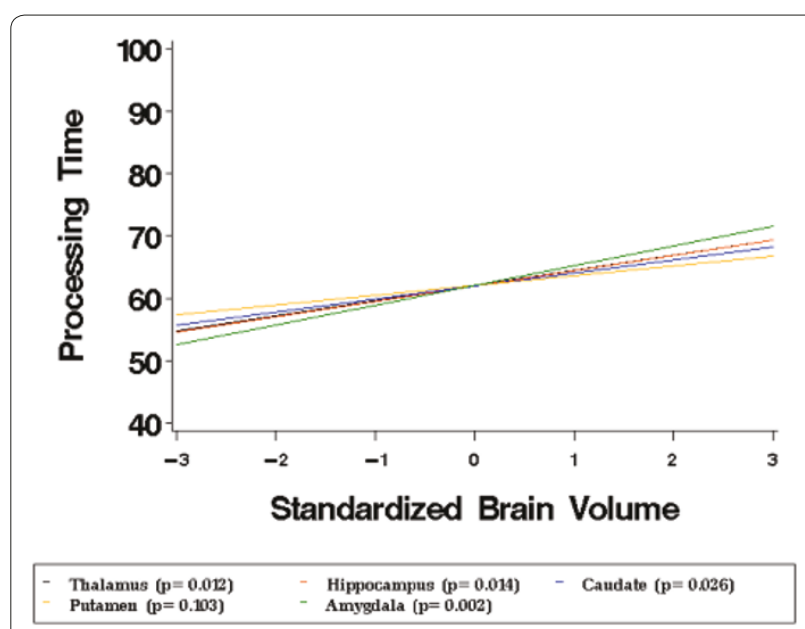

Figure 3. Processing time scores adjusted for age, race, and education are plotted against standardized brain volumes.

The roles of several factors that might influence exposure to head trauma in fighters have been examined. Differences in the type of fighting on volumetric measures were seen. Boxers, in general, had lower thalamic and hippocampal volumes than MMA fighters and had worse scores on diffusion measures. However, both groups showed a negative association between exposure and volume or diffusivity.

On the other hand, in an initial assessment, the weight of the fighter did not influence volumetric results. Specifically, the interaction between weight class and fight exposure did not significantly predict brain volume. For the caudate, there was a trend for an interaction between the number of professional fights and weight class $(P=0.051)$. For lower weight classes, the relationship between reduction in caudate volume with increasing number of fights is greater than for higher weight classes.

There are several limitations to consider in interpreting the cross-sectional findings from the PFBHS. Though all

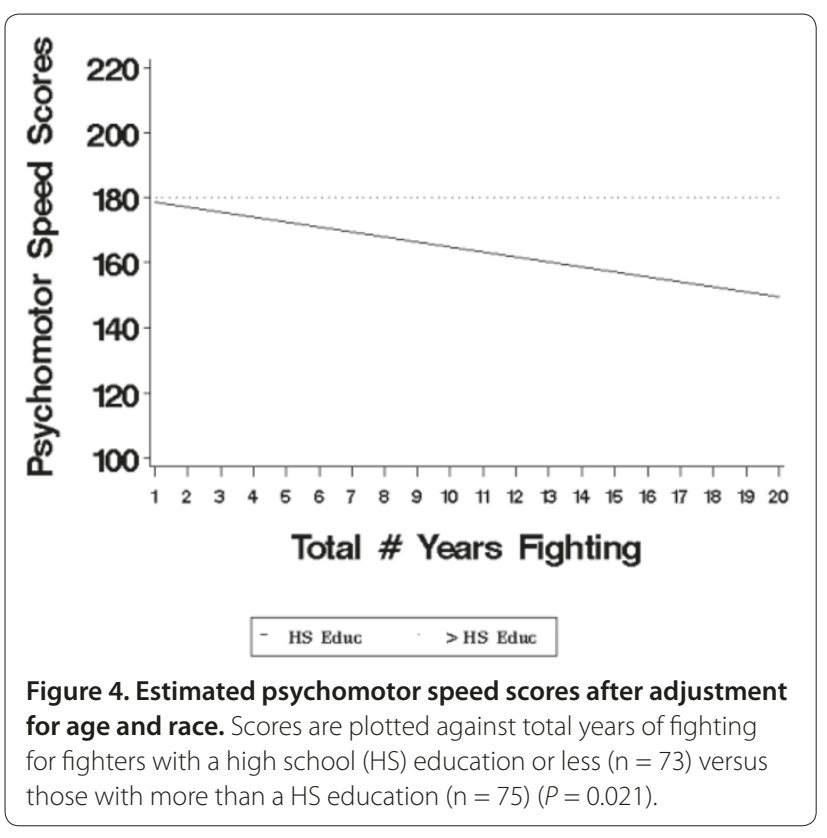

the analyses were adjusted for age and education, we are only now recruiting an age- and education-matched control group, and so we did not have a control group for comparison. Thus, whether the associations between measures of exposure and imaging and clinical outcomes are related to the exposure or other factors is not clear. The study group was not a random sample of fighters; participants were self-selected and may be less skilled or more susceptible to be knocked out. However, in comparison with all those who fought in Nevada over the same year, subjects in the PFBHS differed only in their slightly younger age and their slightly smaller number of professional fights but not in winning percentage or times knocked out. As mentioned above, all of our measures of exposure to head trauma are indirect and may not truly reflect the actual degree of head trauma each subject experienced. 


\section{Conclusions}

It is generally conceded that there is still much work to be done in CTE, understanding its natural history, determining its risk factors, developing diagnostic methods including predictive biomarkers, and ultimately discovering therapeutic measures. Information gathered from epidemiological studies in groups exposed to repetitive head trauma, such as those engaged in combat sports, may guide us in the directions needed to answer the many outstanding questions of CTE. From the established literature on the brain effects of boxing (much of which has design limitations) come a picture of the clinical features of CTE and the recognition that greater exposure to head trauma is associated with increased risk of long-term neurological disease and that a variety of imaging findings can be seen in fighters.

There are a number of large initiatives, either already launched (such as the PFBHS) or in the planning stage, to more rigorously study the effects of repetitive head trauma in both the sports or military arena. Learning from the experience and methods used in investigating other neurodegenerative disease, such as Alzheimer's or Parkinson's disease, we hopefully accelerate our knowledge and treatment of CTE.

This article is part of a series on Traumatic Brain Injury, edited by Robert Stern. Other articles in this series can be found at http://alzres.com/series/traumaticbraininjury

Abbreviations

CTE, chronic traumatic encephalopathy; KO, knockout; MMA, mixed martial arts; MRI, magnetic resonance imaging; PET, positron emission tomography; PFBHS, Professional Fighters Brain Health Study.

\section{Competing interests}

The authors declare that they have no competing interests.

\section{Acknowledgments}

We gratefully acknowledge funding support from the Lincy Foundation. Triny Cooper, Isaac Santa Ana, and Michelle Sholar were all instrumental in this work.

Published: 4 June 2013

\section{References}

1. Martland HA: Punch drunk. J Am Med Assoc 1928, 91:1103-1107.

2. Clausen $\mathrm{H}, \mathrm{McC}$ cory $\mathrm{P}$, Anderson $\mathrm{V}$ : The risk of chronic traumatic brain injury in professional boxing: change in exposure variables over the past century. Br J Sports Med 2005, 39:661-664; discussion 664.

3. Jordan BD: Brain injury in boxing. Clin Sports Med 2009, 28:561-578, vi.

4. Zazryn TR, McCrory RR, Cameron PA: Neurologic injuries in boxing and other combat sports. Neurol Clin 2008, 26:257-227.

5. Mendez MF: The neuropsychiatric aspects of boxing. Int J Psychiatry Med 1995, 25:249-262.

6. Solomon GS, Summer DO, Lovell MR: Long term neurocognitive dysfunction in sports: what is the evidence. Clin Sports Med 2011, 30:165-177.

7. Roberts A: Brain Damage in Boxers: A Study of Prevalence of Traumatic Encephalopathy Among Ex-professional Boxers. London: Pitman Medical Scientific Publishing Co.; 1969.

8. McCrory P, Zazryn T, Cameron P: The evidence for chronic traumatic encephalopathy in boxing. Sports Med 2007, 37:467-476.

9. Heilbronner RL, Bush SS, Ravdin LD, Barth JT, Iverson GL, Ruff RM, Lovell MR, Barr WB, Echemendia RJ, Broshek DK: Neuropsychological consequences of boxing and recommendations to improve safety: a national academy of neuropsychology education paper. Arch Clinical Neuropsychology 2009 24:11-19.

10. Jordan BD: Neurologic aspects of boxing. Arch Neurol 1987, 44:453-459.

11. Jordan BD, Matser EJ, Zimmerman RD, Zazula T: Sparring and cognitive function in professional boxers. Phys Sportsmed 1996, 24:87-98.

12. Mayeux R, Ottman R, Maestre G, Ngai C, Tang MX, Ginsberg H, Chun M, Tycko B, Shelanski M: Synergistic effects of traumatic head injury and apolipoprotein e4 in patients with Alzheimer's disease. Neurology 1995, 45:555-557.

13. Teasdale GM, Nicoll JA, Murray G, Fiddes M: Association of apolipoprotein E polymorphism with outcome after head injury. Lancet 1997, 350:1069-1071.

14. Jordan B: Genetic susceptibility to brain injury in sports: a role for genetic testing in athletes. Phys Sportsmed 1998, 26:25-26.

15. Jordan BD, Relkin NR, Ravdin LD, Jacobs AR, Bennett A, Gandy S: Apolipoprotein E epsilon4 associated with chronic traumatic brain injury in boxing. JAMA 1997, 278:136-140.

16. Corsellis JA, Bruton CJ, Freeman-Browne D: The aftermath of boxing. Psychol Med 1973, 3:270-303.

17. Casson IR, Siegel O, Sham R, Campbell EA, Tarlau M, DiDomenico A: Brain damage in modern boxers. JAMA 1984, 251:2663-2667.

18. Critchley E: Nervous disorders in boxers. Med Annu 1937, 318-320.

19. Guterman A, Smith R: Neurological sequelae of boxing. Sports Med 1987 4:194-210.

20. McKee AC, Stein TD, Nowinski CJ, Stern RA, Daneshvar DH, Alvarez VE, Lee HS, Hall G, Wojtowicz SM, Baugh CM, Riley DO, Kubilus CA, Cormier KA, Jacobs MA, Martin BR, Abraham CR, Ikezu T, Reichard RR, Wolozin BL, Budson AE, Goldstein LE, Kowall NW, Cantu RC: The spectrum of disease in chronic traumatic encephalopathy. Brain 2013, 136:43-64

21. Loosemore M, Knowles CH, Whyte GP: Amateur boxing and risk of chronic traumatic brain injury: systematic review of observational studies. BMJ 2007, 335:809

22. Porter MD: A 9-year controlled prospective neuropsychologic assessment of amateur boxing. Clin J Sport Med 2003, 13:339-352.

23. Moseley LE: The neuroimaging evidence for chronic brain damage due to boxing. Neuroradiology 2000, 42:1-8.

24. Orrison WW, Hanson EH, Alamo T, Watson D, Sharma M, Perkins TG, Tandy RD: Traumatic brain injury: a review and high field MRI findings in 100 unarmed combatants using a literature based checklist approach. J Neurotrauma 2009, 26:689-701.

25. Zhang L, Heier LA, Zimmerman RD, Jordan B, Uluğ AM: Diffusion anisotropy changes in the brains of professional boxers. AJNR Am J Neuroradio/ 2006 27:2000-2004

26. Zhang L, Ravdin LD, Relkin N, Zimmerman RD, Jordan B, Lathan WE, Uluğ AM: Increased diffusion in the brain of professional boxers: a preclinical sign of traumatic brain injury? AJNR Am J Neuroradiol 2003, 24:52-57.

27. Chappell MH, Uluğ AM, Zhang L, Heitger MH, Jordan BD, Zimmerman RD, Watts R: Distribution of microstructural damage in the brains of professional boxers: a diffusion MRI study. J Magn Reson Imaging 2006, 24:537-542.

28. Kemp PM, Houston AS, Macleod MA, Pethybridge RJ: Cerebral perfusion and psychometric testing in military amateur boxers and controls. I Neurol Neurosurg Psychiatry 1995, 59:368-374.

29. Provenzano FA, Jordan B, Tikofsky RS, Saxena C, Van Heertum RL, Ichise M: F-18 FDG PET imaging of chronic traumatic brain injury in boxers: a statistical parametric analysis. Nucl Med Commun 2010, 31:952-957.

30. BoxRec homepage [http://boxrec.com].

31. Mixed Martials Arts LLC homepage [http://mixedmartialarts.com].

32. Sherdog.com homepage [http://sherdog.com]

33. Bernick C, Banks SJ, Obuchowski N, Phillips M, Shin W, Lowe M, Jones S, Modic M: Professional Fighters Brain Health Study: rationale and methods. Am J Epidemiol, in press.

34. Bernick C, Banks S, Mahmoud S, Shin W, Phillips M, Lowe M, Jones S, Modic M: The threshold effect of repeated head trauma on brain structure and cognition: The Professional Fighters Brain Health Study. Neurology 2012, 79:e87-e91.

35. Shin W, Mahmoud S, Sakaie K, Bank S, Lowe M, Phillips M, Modic M, Bernick C Diffusion measures indicate fight exposure-related damage to cerebral white matter in boxers and mixed martial arts fighters. AJNR, in press.

doi:10.1186/alzrt177

Cite this article as: Bernick C, Banks S: What boxing tells us about repetitive head trauma and the brain. Alzheimer's Research \& Therapy 2013, 5:23. 